\title{
AN EXPLICIT CONSTITUTIVE EQUATION FOR PLANE AND AXISYMMETRIC STEADY FLOWS WITH VISCOELASTIC EFFECTS
}

\author{
R. L. Thompson ${ }^{\mathrm{a}}$ \\ and P. R. Souza Mendes ${ }^{b}$ \\ Departamento de Engenharia Mecânica \\ Rua Marquês de São Vicente 225, Gávea \\ CEP. 22453-900, Rio de Janeiro, RJ, Brasil \\ aroney@mec.puc-rio.br \\ bpmendes@mec.puc-rio.br \\ ABSTRACT \\ Non-Newtonian materials respond differently when submitted to shear or extension. A \\ constitutive equation in which the stress is a function of both the rate of deformation and \\ on the type of the flow is proposed and analyzed theoretically. It combines information \\ obtained in shear, extension and rigid body motion in all regions of complex flow. The \\ analysis has shown how to insert some elastic effects in a constitutive equation that depends \\ only on the present time and position. One advantage of the model is that all the steady \\ rheological functions in simple shear flow and in extensional flow are predicted exactly. \\ Another important property that is included is the split of the extensional viscosity in two \\ parts: one dissipative part that is related to the shear viscosity and an elastic part that is \\ related to the first and second normal stress coefficients in shear. A discussion involving \\ the dimensionless numbers that relate elastic and viscosity effects is also given. \\ Keywords: viscoelastic fluid, constitutive model, 2-D flows, flow-type classifier, elastic \\ and dissipative extensional viscosity
}

\section{NOMENCLATURE}

$\begin{array}{ll}\mathbf{A}_{\mathbf{n}} & \text { n-Rivlin-Ericksen tensor } \\ \mathbf{W} & \text { vorticity tensor } \\ \bar{W} & \text { relative rate of rotation tensor } \\ \mathbf{T} & \text { stress tensor } \\ \mathbf{D} & \text { rate-of-strain tensor } \\ \mathbf{L} & \text { transpose of the velocity gradient tensor } \\ \mathbf{v} & \text { velocity vector } \\ \mathbf{C}_{\mathbf{t}}(\tau) & \text { Right-relative-Cauchy-Green-strain tensor } \\ \mathbf{Q} & \text { operator that changes the coordinate system } \\ \mathbf{e}_{\mathbf{i}} & \text { eigenvector of } \mathbf{D} \\ \mathbf{c}_{\mathrm{i}} & \text { coefficient i of a constitutive equation } \\ \mathrm{n} & \text { power-law exponent for a Carreau Model } \\ \mathrm{T}_{\mathbf{R}} & \text { Trouton ratio }\end{array}$

\section{Greek symbols}

$\alpha_{i} \quad$ coefficient $i$ of a constitutive equation

$\hat{a} \quad$ extensional dissipative coefficient

$\gamma \quad$ strain

$\varepsilon \quad$ extensional deformation

$\Psi_{1} \quad$ first normal stress difference

$\Psi_{2} \quad$ second normal stress difference

$\eta \quad$ viscosity

$\lambda \quad$ characteristic time

$\eta_{1} \quad$ first extensional viscosity

$\eta_{2} \quad$ second extensional viscosity

$\rho$ density

$\boldsymbol{\Omega}$ rate of rotation of the eigenvectors of $\boldsymbol{D}$

$\tau \quad$ time of a past event

$\tau$ deviatoric part of the stress tensor

\section{Subscripts}

$\mathrm{u} \quad$ uniaxial extension

$\mathrm{p}$ planar extension

s shear

$0 \quad$ limit of small strain rate

$\infty \quad$ limit of high strain rate

\section{INTRODUCTION}

Some industries such as petroleum, food, cosmetic, have a huge influence on economy. There are a number of processes in these industries in which Non-Newtonian materials are essential components. The need for controlling the rheological properties during these processes is being more and more recognized. Flow simulations are one of the tools to help understanding these problems and to improve efficiency. The choice of the constitutive model to be employed on these simulations is of central importance.

Constitutive equations for non-Newtonian materials can be roughly divided into two categories: one in which the stress tensor is a function of the present time and position and another in which it depends on the past history of the deformation of the material. The first category would give an explicit equation for the stress tensor while the second one needs a differential (or an integral in time) constitutive equation.

In this second group one has to work with a rather complex set of hyperbolic differential 
equations that require approximation methods that numerically preserve the ellipticity of the saddle point problem formed by the momentum and the continuity equations to give stable and accurate numerical solution. This complexity does not necessarily grant excellent predictions for the material functions. One limitation is that they are generally based on a single characteristic time (a relaxation time). For example, the Oldroyd-B model predicts a constant first normal stress coefficient and zero for the second normal stress one. This problem has a worse consequence when calculating flows where a strong elongational component is expected. One good attempt to address this problem is the PTT model as one of its coefficients is more related to shear flows and the other is more related to extensional ones. However, the material functions predicted by this model are not totally independent.

Another feature of constitutive equations is that some have material functions that are inputs of the model while for others the material functions depend on their intrinsic parameters. In this later case, the material functions are obtained by a curve fitting of experimental data but are restricted to the type of function predicted by the constitutive equation. This is an important issue that makes, for example, the GNL a better model than the Lodge-Maxwell when the role of the shear viscosity is the most important in a given problem, because in the former, shear viscosity is a material function given as input.

The present work is a part of a research program which introduces the flow-type classifier proposed by Astarita (1979) in the development of new constitutive equations. The idea was first proposed by Schunk and Scriven (1990) when they proposed a new viscosity function dependent both on the second invariant of $\boldsymbol{D}$, the rate-ofdeformation tensor and on the second invariant of $\overline{\boldsymbol{W}}$, the relative-rate-of-rotation tensor defined by Drout and Lucius (1976). With this assumption, they could fit extensional and shear viscosities separately. Souza Mendes et al. (1995) have further developed this idea by making the stress tensor a function of $\boldsymbol{D}$ and $\overline{\boldsymbol{W}}^{2}$ (two symmetric tensors) and was able to predict non-zero second normal stress coefficient. Thompson et al. have modified the previous assumption by making the stress dependent on the skew-symmetric tensor $\overline{\boldsymbol{W}}$ and could also predict non-zero first normal stress coefficient.
The goal of this paper is to present a new explicit constitutive equation for plane and axisymmetric flows, in which the material functions for simple shear flow and for extensional flow are inputs for the equation and therefore they are predicted exactly. An analysis is given to insert some viscoelastic properties that were present only in constitutive models based on the history of deformation. This is done by choosing how the rheology behavior on the simple limiting kinematic cases, namely, shear, extension and solid body motion, influence complex flows.

\section{THEORETICAL ANALYSIS}

\section{Governing Equations}

The mass equation for incompressible materials is given by:

$$
\nabla \cdot \boldsymbol{v}=0
$$

The momentum equation for steady flow and negligible external forces is:

$$
\rho \mathbf{v} \cdot \nabla \mathbf{v}=\nabla \cdot \mathbf{T}
$$

where $\boldsymbol{T}$, the stress tensor is related to kinematics by the constitutive model that is developed on the present work.

\section{Rivlin-Ericksen Tensors}

The Rivlin-Ericksen tensors are a set of kinematic tensors defined as:

$$
\boldsymbol{A}_{n}(t)=\left.\frac{d^{n}}{d \tau^{n}} \boldsymbol{C}_{t}(\tau)\right|_{\tau=t}, n=0,1,2 \ldots
$$

where $\boldsymbol{C}_{\boldsymbol{t}}(\boldsymbol{\tau})$ is the Right-relative-Cauchy-Greenstrain-tensor, and $\boldsymbol{A}_{\boldsymbol{0}} \equiv \boldsymbol{I}$. Oldroyd has shown that the R-E tensors are related by the following recursive formulae:

$$
A_{n+1}=\dot{A}_{n}+A_{n} \cdot L+L^{T} \cdot A_{n}
$$

where $\boldsymbol{L}$ is the transpose of the velocity gradient. 
For steady flows, one assumption that can be made is that the memory of the viscoelastic material is of instantaneous duration, although it remembers all R-E tensors. This assumption is an alternative to the one that the material has a finite memory which fades in time. Therefore, the extra stress tensor is a function of all the R-E tensors:

$$
\boldsymbol{\tau}=\boldsymbol{\tau}\left(\boldsymbol{A}_{n}\right), \quad n=1,2,3, \ldots
$$

or in a reference frame $R_{f}$ :

$$
\boldsymbol{\tau}=c_{1} \boldsymbol{A}_{1}+c_{2} \boldsymbol{A}_{2}+c_{3} \boldsymbol{A}_{3}+\ldots
$$

where the coefficients $c_{i}$ are functions of the invariants of the R-E tensors. Therefore, this is a generalization of the Retarded-motion-expansion. The model is objective, since all the R-E tensors are. So, if one wants to describe the problem in another reference frame, $R_{f}^{*}$ defined by:

$$
\boldsymbol{e}_{i}^{*}=\boldsymbol{Q} \cdot \boldsymbol{e}_{i}
$$

where $\boldsymbol{Q}$ is an orthonormal tensor. Thus, one would find for the stress tensor:

$$
\boldsymbol{\tau}^{*}=c_{1}^{*} \boldsymbol{A}_{1}^{*}+c_{2}^{*} \boldsymbol{A}_{2}^{*}+c_{3}^{*} \boldsymbol{A}_{3}^{*}+\ldots
$$

where the R-E tensors in this new frame are given by:

$$
A_{n}^{*}=Q \cdot A_{n} \cdot Q^{T}
$$

It is known that the vorticity tensor, the skew-symmetric part of the velocity gradient, has the following relation between the two reference frames:

$$
W^{*}=\dot{Q} \cdot Q^{T}+Q \cdot W \cdot Q^{T}
$$

To continue the analysis it is important to define the tensor $\boldsymbol{\Omega}$, related to the rate of rotation of the eigenvectors of $A_{1}$ :

$$
\dot{\boldsymbol{e}}_{i}=\boldsymbol{\Omega} \cdot \boldsymbol{e}_{i}
$$

From Equations (7) and (11) it is easy to show how the tensor $\boldsymbol{\Omega}^{*}$ in the reference frame $R_{f}^{*}$ is related to $\boldsymbol{\Omega}$ :

$$
\Omega^{*}=\dot{Q} \cdot Q^{T}+Q \cdot \Omega \cdot Q^{T}
$$

Now, it is assumed that there exists a length scale above which the advection of $\boldsymbol{\Omega}$ is very small. Describing the problem in a neighborhood of diameter $l_{1}$ of the material particle of interest, we can change our reference frame to the reference of the eigenvectors of $A_{1}$. The rotation related to the original frame is such that, in this new reference $\boldsymbol{\Omega}^{*}$ vanishes so, $\dot{\boldsymbol{Q}}=-\boldsymbol{Q} \cdot \boldsymbol{\Omega}$ or $\dot{\boldsymbol{Q}}=\boldsymbol{\Omega} \cdot \boldsymbol{Q}^{T}$. Applying this transformation to the vorticity, Eq. (10), we get:

$$
W^{*}=Q \cdot W \cdot Q^{T}-Q \cdot \Omega \cdot Q^{T}=Q \cdot \bar{W} \cdot Q^{T}
$$

where $\overline{\boldsymbol{W}} \equiv \boldsymbol{W}-\boldsymbol{\Omega}$. Now we apply the same transformation to the material derivative of the RE tensors:

$$
\begin{aligned}
& \begin{aligned}
A_{n}^{*}=Q \cdot A_{n} \cdot Q^{T} \Rightarrow & \dot{A}_{n}^{*}=\dot{Q} \cdot A_{n} \cdot Q^{T}+ \\
& +Q \cdot \dot{A}_{n} \cdot Q^{T}+Q \cdot A_{n} \cdot \dot{Q}^{T}
\end{aligned} \\
& \dot{A}_{n}^{*}=Q \cdot\left(\dot{A}_{n}+A_{n} \Omega-\Omega A_{n}\right) \cdot Q^{T}
\end{aligned}
$$

But, from the recursive formulae we have that:

$$
A_{n+1}^{*}=Q \cdot\left(\dot{A}_{n}+A_{n} L-L^{T} A_{n}\right) \cdot Q^{T}
$$

Thus, comparing equations (14) and (15) we can write:

$$
\boldsymbol{A}_{n+1}^{*}=\boldsymbol{Q} \cdot\left(\frac{D^{\Omega}}{D t} \boldsymbol{A}_{n}+\boldsymbol{A}_{n} \cdot(\boldsymbol{L}-\boldsymbol{\Omega})-(\boldsymbol{L}-\boldsymbol{\Omega})^{T} \cdot \boldsymbol{A}_{n}\right) \cdot \boldsymbol{Q}^{T}
$$

where the operator $\frac{D^{\Omega}}{D t} \boldsymbol{M}$ denotes the frame indifferent time derivative of a generic second order tensor $\boldsymbol{M}$, defined as :

$$
\frac{D^{\Omega}}{D t} \boldsymbol{M} \equiv \boldsymbol{M}+\boldsymbol{M} \cdot \boldsymbol{\Omega}-\boldsymbol{\Omega} \cdot \boldsymbol{M}
$$




\section{CIÊNCIA/SCIENCE}

This time derivative is a measure of the variation, following the motion, of the components of the tensor $\boldsymbol{M}$ with respect to the basis of the eigenvectors of $\boldsymbol{D}$. This result shows that:

$$
\boldsymbol{A}_{n+1}=\frac{D^{\Omega}}{D t} \boldsymbol{A}_{n}+\boldsymbol{A}_{n} \cdot(\boldsymbol{D}+\overline{\boldsymbol{W}})-(\boldsymbol{D}-\overline{\boldsymbol{W}}) \cdot \boldsymbol{A}_{n}(18
$$

By induction, it can be shown that if:

$$
\frac{D^{\Omega}}{D t} \boldsymbol{A}_{\boldsymbol{n}}=\Phi\left(\boldsymbol{A}_{i}, \overline{\boldsymbol{W}}\right) \quad i=1 \ldots n
$$

equation (6) would reduce to $\boldsymbol{\tau}=\boldsymbol{\tau}(\boldsymbol{D}, \overline{\boldsymbol{W}})$. An interesting thing to notice is that for $\frac{D^{\Omega}}{D t} \boldsymbol{A}_{\boldsymbol{n}} \equiv \boldsymbol{0}$, than is the solution of the Zorowaski problem and the flow is steady in the frame of the eigenvectors of. This particular case is a MWCSH (Motion with Constant Stretch History).

\section{The Constitutive Equation}

It can be shown that a symmetric tensorvalued isotropic function of a symmetric $(\boldsymbol{D})$ and a skew-symmetric $(\overline{\boldsymbol{W}})$ tensor has the following general form:

$$
\begin{aligned}
\boldsymbol{T} & =\bar{\alpha}_{0} \boldsymbol{I}+\bar{\alpha}_{1} \boldsymbol{D}+\bar{\alpha}_{2} \overline{\boldsymbol{W}}^{2}+\alpha_{3} \boldsymbol{D}^{3}+ \\
& +\bar{\alpha}_{4}(\boldsymbol{D} \overline{\boldsymbol{W}}-\overline{\boldsymbol{W}} \boldsymbol{D})+\overline{\alpha_{5}} \overline{\boldsymbol{W}} \boldsymbol{D} \overline{\boldsymbol{W}}+ \\
& +\bar{\alpha}_{6}\left(\boldsymbol{D}^{2} \overline{\boldsymbol{W}}-\overline{\boldsymbol{W}} \boldsymbol{D}^{2}\right)+ \\
& +\bar{\alpha}_{7}\left(\overline{\boldsymbol{W}} \boldsymbol{D} \overline{\boldsymbol{W}}^{2}-\overline{\boldsymbol{W}}^{2} \boldsymbol{D} \overline{\boldsymbol{W}}\right)
\end{aligned}
$$

where the coefficients $\alpha_{i}$ are functions of scalar invariants:

$$
\begin{aligned}
& \bar{\alpha}_{i}={\overline{\alpha_{i}}}_{i}\left(\operatorname{tr} \boldsymbol{D}, \operatorname{tr} \boldsymbol{D}^{2}, \operatorname{tr} \boldsymbol{D}^{3}, \operatorname{tr} \overline{\boldsymbol{W}}^{2}, \operatorname{tr} \boldsymbol{D} \overline{\boldsymbol{W}}^{2}\right. \\
& \left.\operatorname{tr} \boldsymbol{D}^{2} \overline{\boldsymbol{W}}^{2}, \operatorname{tr} \boldsymbol{D}^{2} \overline{\boldsymbol{W}}^{2} \boldsymbol{D} \overline{\boldsymbol{W}}\right)
\end{aligned}
$$

\section{Simplifications for 2-D Incompressible Flows}

\section{PLANE FLOWS}

Equation (20) is significantly simplified for plane incompressible flows (Pope, 1975; has done this for $\boldsymbol{W}$ in the place of $\overline{\boldsymbol{W}}$.

Tensor $\overline{\boldsymbol{W}}$ can be expressed as a function of $\boldsymbol{D}^{2}$, $\operatorname{tr} \overline{\boldsymbol{W}}^{2}$ and $\operatorname{tr} \boldsymbol{D}^{2}$.

Tensor $\overline{\boldsymbol{W}} \boldsymbol{D} \overline{\boldsymbol{W}}=-\frac{1}{2}\left(\operatorname{tr} \overline{\boldsymbol{W}}^{2}\right) \boldsymbol{D}$

Tensor $\boldsymbol{D}^{2} \overline{\boldsymbol{W}}-\overline{\boldsymbol{W}} \boldsymbol{D}^{2}=\mathbf{0}$

Tensor $\overline{\boldsymbol{W}} \boldsymbol{D} \overline{\boldsymbol{W}}^{2}-\overline{\boldsymbol{W}}^{2} \boldsymbol{D} \overline{\boldsymbol{W}}=-\frac{1}{2}\left(\operatorname{tr} \overline{\boldsymbol{W}}^{2}\right)(\boldsymbol{D} \overline{\boldsymbol{W}}-\overline{\boldsymbol{W}} \boldsymbol{D})$

Furthermore,

$$
\operatorname{tr} \boldsymbol{D}=\operatorname{tr} \boldsymbol{D}^{3}=\operatorname{tr} \boldsymbol{D} \overline{\boldsymbol{W}}^{2}=\operatorname{tr} \boldsymbol{D}^{2} \overline{\boldsymbol{W}}^{2} \boldsymbol{D} \overline{\boldsymbol{W}}=0
$$

Hence, equations (20) and (21) and reduce to:

$$
\begin{gathered}
\boldsymbol{T}=\alpha_{0} \boldsymbol{I}+\alpha_{1} \boldsymbol{D}+\alpha_{3} \boldsymbol{D}^{2}+\alpha_{4}(\boldsymbol{D} \overline{\boldsymbol{W}}-\overline{\boldsymbol{W} D}) \\
\bar{\alpha}_{i}=\bar{\alpha}_{i}\left(\operatorname{tr} \boldsymbol{D}^{2}, \operatorname{tr} \overline{\boldsymbol{W}}^{2}\right)
\end{gathered}
$$

\section{AXISYMETRIC FLOWS}

If we considered axisymmetric incompressible flows, however, the only simplification is the following

$$
\overline{\boldsymbol{W}} \boldsymbol{D} \overline{\boldsymbol{W}}^{2}-\overline{\boldsymbol{W}}^{2} \boldsymbol{D} \overline{\boldsymbol{W}}=-\frac{1}{2}\left(\operatorname{tr} \overline{\boldsymbol{W}}^{2}\right)(\boldsymbol{D} \overline{\boldsymbol{W}}-\overline{\boldsymbol{W} D}) .
$$

\section{Performance in Shear and Extension}

Every viscometric flow is locally a simple shear flow. For simple shear flow $\left(v=\dot{x}_{2} \boldsymbol{e}_{1}\right.$, where $\gamma$ is the shear rate), Eq. (23) gives the following expressions for the material functions:

$$
\begin{aligned}
& \eta_{s} \equiv \frac{2}{\dot{\gamma}}=\frac{T_{21}}{2} \alpha_{1} \\
& \psi_{1} \equiv \frac{T_{11}-T_{22}}{\dot{\gamma}^{2}}=\alpha_{4} \\
& \psi_{2} \equiv \frac{T_{22}-T_{33}}{\dot{\gamma}^{2}}=\frac{1}{4}\left(\alpha_{3}-2 \alpha_{4}\right)
\end{aligned}
$$


CIÊNCIA/SCIENCE

For extensional

flows $\boldsymbol{v}=\dot{\varepsilon} x_{1} \boldsymbol{e}_{1}-\frac{1}{2} \dot{\varepsilon}(1+b) x_{2} \boldsymbol{e}_{2}-\frac{1}{2} \dot{\varepsilon}(1-b) x_{3} \boldsymbol{e}_{3}$ where $0 \leq b \leq 1$ and $\dot{e}$ is the extension rate, Eq. (23) gives the following expressions for the material functions:

$$
\begin{aligned}
& \bar{\eta}_{1} \equiv \frac{T_{11}-T_{22}}{\dot{\varepsilon}}=\frac{3+b}{2} \alpha_{1}+\frac{4-(1+b)^{2}}{4} \alpha_{3} \dot{\varepsilon} \\
& \bar{\eta}_{2} \equiv \frac{T_{33}-T_{22}}{\dot{\varepsilon}}=b \alpha_{1}+b \alpha_{3} \dot{\varepsilon}
\end{aligned}
$$

Extensional flows, have three possible cases for plane and axisymmetric geometries: planar elongation $(b=1)$, uniaxial elongation $(\dot{\varepsilon}>0$ and $b=0)$ and biaxial stretching $(\dot{\varepsilon}<0$ and $b=0)$. For the first case, the material functions are given by:

$$
\begin{aligned}
& \bar{\eta}_{1} \equiv \frac{T_{11}-T_{22}}{\dot{\varepsilon}}=2 \alpha_{1} \\
& \bar{\eta}_{2} \equiv \frac{T_{33}-T_{22}}{\dot{\varepsilon}}=\alpha_{1}+\alpha_{3} \dot{\varepsilon}
\end{aligned}
$$

For the second case, the second viscosity vanishes and we can define a function $\eta_{u}$ as onethird of the extensional viscosity:

$$
\left.\eta_{u} \equiv \frac{T_{11}-T_{22}}{3 \dot{\varepsilon}}\right|_{b=0}=\frac{1}{2} \alpha_{1}+\frac{1}{4} \alpha_{3} \dot{\varepsilon}
$$

All steady material functions are defined with the deformation rate as the independent variable. Another variable is needed to classify the type of the flow. With these two variables, using Eq. (23) we can obtain (25) to (32) independently. So, for this reason, the result concerning the dependence on the scalar invariants (24), obtained for plane flows, are extended to axisymmetric flows. Therefore it will be considered that for plane and axisymmetric flows,

or in an equivalent form that shows explicitly the idea at the beginning of the paragraph:

$$
\alpha_{i}=\alpha_{i}(\dot{\gamma}, R)
$$

where $\dot{\gamma} \equiv\left(2 t r D^{2}\right)^{\frac{1}{2}}$ is the deformation rate and $R \equiv-\frac{t r D^{2}}{t r \bar{W}^{2}}$ is a flow-type classifier.

\section{Flow-type Classifier}

In order to characterize the flow, the criterium presented in Astarita (1979), based on the invariance of the relative-rate-of-rotation tensor, is used here. The deformation rate parameter $\left(s^{2}\right)$ and the rotation rate parameter $\left(\omega^{2}\right)$ are defined respectively as:

$$
s^{2}=\operatorname{tr}\left(S^{2}\right)=S_{m n} S_{n m}, w^{2}=-\operatorname{tr}\left(\bar{W}^{2}\right)=-\bar{W}_{m n} \bar{W}_{n m}
$$

Defining $R$ as the ratio between these two quantities:

$$
R=\frac{\omega^{2}}{s^{2}}=-\frac{\bar{W}_{m n} \bar{W}_{n m}}{S_{m n} S_{n m}}
$$

which take values from 0 to $\infty$.

This kind of parameter can be used to classify flows. It is local, objective, is not restricted to a class of flow and is entirely kinematic (there is no material property involved). It is a measure of how much the material avoids stretching by means of a rotation (relative to the principal directions of stretching). In this sense, it is expected that at one side of the limiting cases stands the extensional kinematics, because the material is not avoiding to be stretched at all; while at the other side is the rigid body motion, when the material is not stretched. In fact, it can be shown (Astarita, 1979) that for pure extensional flow, $R=0$ and as the flow approaches to a solid body motion, $R \rightarrow \infty$. Every other kind of flow lies in between, including pure shear flows, where $R=1$, which is considered to be another limiting case (on a 2-D Cartesian flow the value $R=1$ is the supremum for elliptical flows (Astarita, 1991), and a infimum for strong flows, (Tanner, 1975). This parameter was firstly used in constitutive models first in Schunk and Scriven (1990), where extensional and shear viscosity effects were decoupled; in Paulo et al (1995) where a new weight function for the 
viscosity was proposed and the prediction for the second normal stress difference was included and in Thompson et al. (1999), where all the rheological functions for stationary flow, in shear (including the first normal stress difference) and extension are being carried to regions of complex flows.

This quantity can be normalized to avoid numerical problems when $R \rightarrow \infty$ :

$$
D=\frac{1-R}{1+R}
$$

The scalar $D$ will take values between -1 and +1 . For pure shear flow, $D=0$; for pure extensional flow, $D=+1$ and as the flow approximates to a solid body motion, $D \rightarrow-1$. With this map that places the type of kinematics that the fluid is going through an excellent tool for the interpretation of results is provided.

\section{Second Law of Thermodynamics}

\section{Rate of work done by the stress tensor}

The rate of work done by the stress tensor that contributes to the change of internal energy is $\boldsymbol{T}: \nabla \boldsymbol{v}$. When $\boldsymbol{T}$ is given by Eq. (23), this quantity can be written as:

$$
\boldsymbol{T}: \nabla \boldsymbol{v}=\operatorname{tr}\left[\alpha_{1} \boldsymbol{D}^{2}+\alpha_{3} \boldsymbol{D}^{3}\right]
$$

For a GNL fluid, second law of thermodynamics would require that $\boldsymbol{T}: \nabla \boldsymbol{v} \geq 0$. This is equivalent to assuming that the rate of work is always dissipative. This happens because a GNL fluid is a substance whose thermodynamic state is defined by two intensive thermodynamic properties. It can not predict any elastic material function or behavior. Since we can make any tensor between $\boldsymbol{D}^{2}$ and $\boldsymbol{D}^{3}$ dominant, this condition would result in:

$$
\begin{aligned}
& \operatorname{tr}\left[\alpha_{1} \boldsymbol{D}^{2}\right] \geq 0 \\
& \operatorname{tr}\left[\alpha_{3} \boldsymbol{D}^{3}\right] \geq 0
\end{aligned}
$$

Some conclusions would be that $\alpha_{1} \geq 0$, and $\alpha_{3}$ has the opposite sign of $\operatorname{tr} \boldsymbol{D}^{3}$. These restrictions do not necessarily apply to the present model, despite the fact that the stress is a sole function of the present time and position. For a perfect elastic material, if we have uniaxial elongation, it is expected that this material will store energy, and for a biaxial stretching, its internal energy would decrease (in this case, this would require that $\alpha_{3} \geq 0$ ). For a viscoelastic material a dissipative and an elastic rate of work exists together. It can be stretched (increasing its internal energy) without changes on density and temperature. For an incompressible material and isothermal flow, the second law of thermodynamics becomes (Mackay and Astarita, 1996):

$$
\boldsymbol{T}: \nabla \boldsymbol{v} \geq \rho \frac{D a}{D t}
$$

where $a$ is the Helmholtz free energy. Therefore, the rate of Helmholtz free energy indicates the elastic part of the stress power. For a perfect elastic material, Eq. (40) holds as an equality. For a GNL fluid, the right side vanishes.

Another important observation lies on one of the main differences between simple shear flow and extensional flow. In simple shear, normal stresses are decoupled from viscous stresses. First and second normal stresses differences in shear are strictly related to elastic effects while shear viscosity is dissipative. On extensional flow there are only normal stresses and therefore on its material functions there are dissipative and elastic effects coexisting. So the change of a viscoelastic material from anextensional kinematic to simple shear can be seen as a filter that separates dissipative from elastic espectra. Therefore, it does not seem appropriate to use, for the normal stresses differences in extension, the term "extensional viscosities". The origin of the name is probably related to the Newtonian fluid, where there is no elasticity and therefore extensional material functions involve the shear viscosity only. One way of formulating this idea (see Thompson, 2001) is to separate elastic and dissipative effects of extensional viscosity by using a parameter $\beta$ $(0 \leq \beta \leq 1)$, the extensional dissipative coefficient: 


$$
\eta_{u}=\left(\eta_{u}\right)_{\text {diss }}+\left(\eta_{u}\right)_{\text {elast }}=\beta \eta_{u}+(1-\beta) \eta_{u}
$$

One case of interest, for viscoelastic materials, is when the dissipative part of the extensional viscosity (for axisymmetric flows) is exactly the shear viscosity. Defining $\bar{T}_{R}$ as onethird of the usual Trouton ratio, the extensional dissipative coefficient is:

$$
\beta=\frac{1}{\bar{T}_{R}}
$$

Consequently, the elastic part of the extensional viscosity is:

$$
\left(\eta_{u}\right)_{\text {elast }}=\frac{\bar{T}_{R}-1}{\eta_{s}}
$$

This assumption (43) relates the Trouton ratio with the elasticity of the fluid. The higher is the Trouton ratio, the more elastic is the fluid as the literature reports. For GNL fluids, $\bar{T}_{R}=1 \Rightarrow \beta=1$ and $\left(\eta_{u}\right)_{\text {elast }}=0$, which means that the extensional viscosity is totally dissipative.

Because of the difficulty in reaching steady state in many extensional flows of polymeric liquids, transient data are very important. Bird (1987) reports some measurements made by Meissner (1984) with a polyisobutylene at $T=296 K$ on the elongational stress growth function $\bar{\eta}^{+}$with respect to time. These data are normalized as follows: for planar elongation, the data for $\bar{\eta}_{1}^{+}$is divided by $4, \bar{\eta}_{2}^{+}$is divided by 2 and for uniaxial elongation and biaxial stretching, $\bar{\eta}^{+}$ is divided by 3 . This is done so that the sets should equal $\eta^{+}$, the shear stress growth function in the linear viscoelastic limit. These results are in accordance with that assumption. For small $\dot{\varepsilon}, \bar{\eta}_{u}^{+}$ is a little bit higher for uniaxial elongation then $\bar{\eta}^{+}$and for biaxial stretching $\bar{\eta}_{u}^{+}$is a little bit lower then $\bar{\eta}^{+}$, (see Eq. (32)). For plane flows, $\frac{\bar{\eta}_{1}^{+}}{4}$ have the same behavior of $\bar{\eta}^{+}$and $\frac{\bar{\eta}_{2}^{+}}{2}$ is lower then $\bar{\eta}^{+}$(see Eqs. (30) and (31)).

Another case of interest is when nonspheric rigid particles are added to a Newtonian solvent. There are cases in which this procedure would result in a difference of the shear behavior making the fluid shear-thinning without changing significantly the extensional viscosity. In this case $\bar{T}_{R}>1$ does not imply the presence of elastic effects. This is a complex behavior to model. The problem is that simple models predict similar behavior between extensional and shear viscosities while complex models predict different behavior due to elasticity. The present model is able to predict different behavior between these quantities in inelastic fluids.

\subsection{The $\alpha_{i}$ functions}

On Thompson (2001) there is detailed information about the choices for the $\alpha_{1}$ functions. The coefficient $\alpha_{0}$ is such that makes the extrastress tensor deviatoric, or:

$$
\alpha_{0}=-p-\frac{1}{3} \alpha_{3} \operatorname{tr} D^{2}
$$

Examination of Equations (25) to (32) indicates that coefficient $\alpha_{1}$ is related to dissipative phenomena while $\alpha_{3}$ and $\alpha_{4}$ are related to elastic effects.

The coefficient $\alpha_{1}$ is multiplying $\boldsymbol{D}$ and therefore it has a strong relation with viscosity. For simple shear flows is the shear viscosity and for extensional flows is related to the extensional viscosity. Schunk and Scriven (1990), proposed a viscosity function that was the arithmetic mean between that two viscosities. As generally $T_{R} \geq 3$, this formulation would give a negative viscosity for flows in which $1 \leq R \leq \infty$ and they included the condition $R \leq 1$. Astarita (1991), examining streamline elliptical flows concluded that for a Maxwell fluid the viscosity function decreases with the increase of deformation rate. An assumption 


\section{CIÊNCIA/SCIENCE}

of a geometric mean between the two viscosities (in shear and extension) and the permission of an extrapolation to values of $R$ higher than 1 would give to the present formulation that feature. Another thing to notice is that an interesting property of the geometric mean is its indifference to a change of variable $y=\frac{1}{x}$ or:

$$
G=\sqrt{a b} \Rightarrow \frac{1}{G}=\sqrt{\frac{1}{a} \frac{1}{b}}
$$

This means that had we written momentum equation based on the fluidity instead of the viscosity, we would find the same final result for the property for intermediate values of (this is not true for the arithmetic mean). Based on the ideas presented in the last section, in the present work is proposed a generalization of the viscosity function proposed by Souza Mendes et al. (1995). It makes the coupling between shear viscosity and extensional dissipative part of extensional viscosity. It is a "dissipative function":

For plane flows:

$$
\alpha_{1}=2 \eta_{s}^{R}\left(\eta_{p}\right)^{1-R}
$$

where $\eta_{p}=\frac{1}{4} \bar{\eta}_{1}$

For axisymmetric flows:

$$
\alpha_{1}=2 \eta_{s}^{R}\left(\beta \eta_{u}\right)^{1-R}
$$

The coefficient $\alpha_{3}$ makes the coupling between shear and extensional elastic effects. The geometric mean would give a formulation in which there is no possibility for a material to have an elastic behavior in one type of flow and a nonelastic behavior on the other, what is physically reasonable:

For plane flows:

$$
\alpha_{3}=\left(2 \psi_{1}+4 \psi_{2}\right)^{R}\left[\frac{\frac{1}{2} \overline{\eta_{1}}-\overline{\eta_{2}}}{\dot{\varepsilon}}\right]^{1-R}
$$

For axisymmetric flows:

$$
\alpha_{3}=\left(2 \psi_{1}+4 \psi_{2}\right)^{R}\left[\frac{4(1-\beta) \eta_{u}}{\dot{\varepsilon}}\right]^{1-R}
$$

However, the arithmetic mean gives the possibility to study shear and extensional elastic effects separately:

For plane flows:

$$
\alpha_{3}=\left(2 \psi_{1}+4 \psi_{2}\right) R+\left[\frac{\frac{1}{2} \overline{\eta_{1}}-\overline{\eta_{2}}}{\varepsilon}\right](1-R)
$$

For axisymmetric flows:

$$
\alpha_{3}=\left(2 \psi_{1}+4 \psi_{2}\right) R+\left[\frac{4(1-\beta) \eta_{u}}{\dot{\varepsilon}}\right](1-R)
$$

The only information that we have for the coefficient $\alpha_{4}$ is that, in shear, it is equal to the first normal stress difference coefficient. It is given by:

$$
\alpha_{4}=\alpha_{4}^{1-R} \psi_{1}
$$

\section{Dimensionless Relations Between Elasticity and Viscosity}

Next step is to adopt an equation to fit extensional and shear rheological data. For shear viscosity one can model as a "power-law" fluid, or a viscoplastic model such as Bigham or Herschel Bulkley, etc. Carreau model is a choice common in literature for viscoelastic materials. So the viscosities are represented by the following equations:

$$
\begin{aligned}
& \frac{\eta_{s}-\eta_{s}^{\infty}}{\eta_{0}-\eta_{s}^{\infty}}=\left[1+\left(\lambda_{s} \dot{\gamma}\right)^{2}\right]^{\frac{\eta_{s}-1}{2}} \\
& \frac{\eta_{u}-\eta_{u}^{\infty}}{\eta_{0}-\eta_{u}^{\infty}}=\left[1+\left(\lambda_{u} \dot{\gamma}\right)^{2}\right]^{\frac{\eta_{u}-1}{2}}
\end{aligned}
$$

where $\eta_{0}$ viscosity plateau for shear and the 
normalized extensional viscosity when $\dot{\gamma} \rightarrow 0$.

$\lambda_{s}$ e $\lambda_{u}$ are characteristic times of the fluid in shear and extensional flow respectively. The parameters $\eta_{s}\left(0<\eta_{s} \leq 1\right)$ and $\eta_{u}\left(0<\eta_{u} \leq 1\right)$ are the powerlaw exponents. If $\eta_{s}=\eta_{u}=1$, then the fluid is Newtonian. Shear-thinning and extensionalthickening behavior (common in viscoelastic fluids) is attained for $\eta_{0}-\eta_{s}^{\infty}>0$ and $\eta_{0}-\eta_{u}^{\infty}<0$. Even in this case, one could model a Boger fluid by imposing $\eta_{0}-\eta_{s}^{\infty}=0$. For the mentioned case $\lambda=\lambda_{u}=\lambda_{s}$ would be an extensional characteristic time, since it would be of no influence in shear. There are some experiments that indicate that the characteristic times and exponents are equal for the same fluid. Analyzing the time spectrum of the fluid, one could choose the higher value to input in a constitutive equation. It is a good assumption to say that complex flows are govern by more than one characteristic time. The consequence of modelling a fluid with only one characteristic time, is a lack of the prediction capability of general phenomena. To increase the capability of prediction of complex phenomena, on the present work it is proposed two different dimensionless numbers relating elastic and viscous effects: one based on shear and the other on extensional parameters. Both consist of a product $\lambda \dot{\gamma}$. The first is a Deborah number, in which the characteristic time of the fluid is, as usual, based on the ratio between two shear functions when $\dot{\gamma} \rightarrow 0: \lambda=\frac{\psi_{10}}{2 \eta_{s 0}}$. The other can be called a Weissenberg number and is based on the extensional characteristic time of the Carreau equation.

\section{FINAL REMARKS}

We have presented a theoretical discussion about a new constitutive equation, which is based on the assumption that the stress tensor is a function of both the rate-of rotation-tensor and the relativerate-of-rotation tensor $(\boldsymbol{T}=\boldsymbol{T}(\boldsymbol{D}, \overline{\boldsymbol{W}}))$. We have discussed the relation between this assumption and the assumption used to develop the retarded motion expansions, namely, that the extra-stress is a function of the Rivlin-Ericksen tensors. We showed that for plane flows the coefficients arising in the representation theorem for $\boldsymbol{T}=\boldsymbol{T}(\boldsymbol{D}, \overline{\boldsymbol{W}})$, the $\alpha_{i}$ 's, are scale functions of the deformation rate, $\dot{\gamma}$, and of the flow-type classifier, $R$. Finally, we analyze the $\alpha_{i}$ coefficients in light of the second law of thermodynamics, and propose to split the extensional viscosity into two additive terms, namely, a dissipative term and an elastic term.

\section{ACKNOWLEDGEMENTS}

This work was funded by grants from FAPERJ.

\section{REFERENCES}

Astarita, G., 1979, Objective and generally applicable criteria for flow classification, Journal of Non-Newtonian Fluid Mechanics, Vol. 6, pp 69-76.

Astarita, G., 1991, Quasi-Newtonian constitutive equations exhibiting flow-type sensitivity, Journal of Rheolgy, Vol. 35(4), pp. 687-689.

Bird, R. B., Armstrong, R. C., and Hassager, O., 1987, Dynamics of polymeric liquids, John Wiley \& Sons, New York, USA, 650p.

Meissner, J., 1984, Pure and Applied Chem., Vol.56, pp. 369-384.

Schunk, P.R. and Sriven, L.E. Constitutive equation for modeling mixed extension and shear in polymer solution processing Journal of Rheology, Vol. 34(7), pp. 1085-1117.

Souza Mendes, P. R., Padmanabhan, M., Scriven and Makosco, C. W., 1995, Inelastic constitutive equation for Non-Newtonian , Rheologica Acta, Vol.(34), pp. 209-214.

Tanner, R. I. and Huilgol, R. R., 1975, On the classification scheme for flow fields, Rheologica Acta, Vol.(14), pp. 959-961.

Thompson, R. L., 2001, Performance of a new constitutive equation for Non-Newtonian liquids (in Portuguese), Ph. D. thesis, Pontifícia Universidade Católica.

Thompson, R. L., Souza Mendes, P. R., and Naccache, M. F., 1999, A new constitutive equation and its performance in contraction flows, Journal of Non-Newtonian Fluid Mechanics, Vol. 86, pp. 375-388. 